\title{
Lossless JPEG-Huffman Model for Digital Image Compression
}

\author{
Gabriel Babatunde Iwasokun \\ Department of Software Engineering \\ Federal University of Technology, Akure, Nigeria \\ gbiwasokun@futa.edu.ng
}

\begin{abstract}
The transmission of digital images has been bedevilled with limitation of storage and bandwidth capacities. One of the common strategies to resolving this limitation is to perform pre-transmission compression on the images. In this research, a lossless Joint Photography Expert Group (JPEG) and Huffman algorithms-based model for digital image compression is proposed. The lossless JPEG component of the model was used to perform Differential Pulse Coding Modulation (DPCM) on the pixels while adaptive Huffman coding was used for quality improvement and standardization. The implementation was carried out in an environment characterized by Windows 10 with Visual Basic as frontend on Personal Computer with 4 GB RAM, 500 GB ROM and 2.2 Ghz Core i3 Processor. The experimental images used for testing the algorithms were acquired from Signal and Image Processing Institute in the University of Southern California (USC-SIPI). Camera (Nikon D7000) and Geographical Information System (GIS) images were also used. Quantitative analyses of the experimental results and performance evaluation using Compression Ratio (CR), Bits per pixel (Bpp), Maximum Difference (MD), Mean Square Error (MSE), Root Mean Square Error (RMSE), Peak Signal Noise Ratio (PSNR), Average Difference (AD) and Structural Content (SC) were carried out. The analyses showed good compression rates and ratios for the proposed model. The superiority of the integrated model over some existing and related ones is also established.
\end{abstract}

Keywords: Digital image, image compression, lossless JPEG, Huffman algorithm, GIS

\section{Introduction}

An image is a two dimensional visual signal of any object captured by optical devices for processing, storage, transformation or transmission by computer applications. It is also described as an artefact which displays visual perception in $\mathrm{x}$ and $\mathrm{y}$ spatial coordinates based on numeric representation of a two-dimensional function $f(x, y)$ over a give amplitude[1-2]. For a digital image, the spatial coordinates and the amplitude are all finite and discrete [3]. A digital image requires enormous storage due to its continuous tone and its storage can be in any form or representation with the existence of diverse conversion algorithms [4]. Digital images are classified into raster and vector. Raster images are built via a finite set of digital values, known as picture elements or pixels which represent the atomic elements and colour equivalent in the image. Electronic device-based images are notable examples of raster images. Vector images possess magnitudes and directions which are often derived from mathematical calculations. This type of images is often required when accuracy and precision are of necessity [5]. Image processing involves carrying out selected or pre-determined operations which include enhancement, restoration, segmentation, representation and compression [6-10]. Image compression is a storage optimization strategy which involves the reduction of data through 
Gabriel Babatunde I wasokun; Lossless JPEG-Huffman Model for Digital Image Compression. Advances in Image and Video Processing, Volume 7 No 1, February (2019); pp: 1-12

elimination of all redundancies for acceptable quality and representation. Compression also fosters speedy transmission of images in channels with limited bandwidth [11-12]. Specifically, image compression is used in the elimination of psycho-visual, inter-pixel and coding redundancies in medical image processing, space imagery, web design, email attachment among others [13]. Image compression can be via lossy or lossless method. Lossy compression uses inexact approximations (or partial data discarding) for representing the content being encoded and it is mostly used for reduction of image data level prior to storage, handling and transmission [14-16]. The two basic lossy compression schemes are lossy transform codecs and lossy predictive codecs. While the former takes samples of images, chopped them into small segments and transform into a new and quantized space, the later is used to predict the image frame followed by quantization and coding of the error between the predicted and the real data, together with any extra information needed for reproducing the prediction [17]. This paper presents the integration of lossless JPEG and Huffman algorithms for digital image compression as solution to some of the existing problems and challenges of digital image compression. Section 2 presents the synopsis of some related works while Sections 3 and 4 present the proposed model and the experimental study respectively. The conclusion drawn from the research is presented in Section 5 .

\section{Related Works}

Numerous research works exist for digital image compression using lossy and lossless techniques. In [18], an enhanced Lempel-Ziv-Welch (LZW) lossless compression technique for medical images is presented. The research sought improvement for some lossless compression techniques and attempted to provide a compression technique for all image formats. The resultant technique is however computationally expensive and speed deficient. The authors in [19] presented a hybrid transform technique as a solution to the problem of large storage requirements and transmission bandwidths of uncompressed images. The research used Discrete Cosine Transform (DCT) and Discrete Wavelet Transform (DWT) based model for quality preserved compression of reconstructed images. Though experimental results on sufficiency of the model for accomplishing reasonable compression and decompression were presented, the proposed model is computationally expensive and underperforms with severally corrupted images. The authors in [15] presented a lossless image compression technique using combinatorial methods. The research focused on improving the effectiveness of Bose Chaudhuri and Hoc-quenghen (BCH) and LZW algorithms. However, the resultant algorithm is susceptible to low compression ratio and its efficiency with large data set not verified. Motivated by the need to develop a lossless decomposition algorithm which is independent of the nature and pattern of text, the authors in [20], developed a lossless text compression technique using ambigram logic and Huffman coding. The technique only focuses on text data compression without any impact on digital images. A lossless compression method based on run-length and LZW coding for digital image disk space requirement minimization is presented in [21]. The method offered an efficient means of compressing images without loss of information during transmission but its operational speed is low. A Huffman coding lossless method for image compression and decompression is presented in [22]. The method reduced the amount of disks space requirement for digital image storage but requires high speed devices for reasonable performance.

In [23], a fast and efficient lossless method for digital image compression is presented. The method implemented single bits coding, binary codes and Golomb codes for the estimation of parameters. The method attained improved compression speed but can be faulty with accidental use of contents with flat probability. The authors in [24] presented an arithmetic entropy coder based predictor model for 
compression of medical images. The model used context-based adaptive lossless image coding scheme for encoding and decoding in raster scan order based on prior scan lines of coded pixels for prediction and text formation. The predictor model is limited by its reliance on image smoothness assumption and mandatory selection of binary or continuous-tone mode. In [25], a lossless digital image compression technique based on simple arithmetic operations and Xilinx Field Programmable Gate Array (FPGA) is proposed. The technique is most applicable for medical and scientific images where lossy compression is not practicable. The technique requires very complex hardware for implementation and also performs poorly for low quality images. The authors in [26] presented a throughput efficient digital image compression technique that is based on Set Partitioning in Hierarchical Trees (SPIHT) algorithm. The SPIHT algorithm was used to eliminate the inherent redundancies among wavelet coefficients as well as the dynamic data structures which hinder hardware realizations. The technique successfully preserves and presents significant information by interchanging the sorting and refinement passes.

\section{Proposed Model}

The proposed model was motivated by the need to address some of the aforementioned limitations and its architecture is represented in the Figure 1 showing a combination of lossless JPEG and Huffman algorithms for digital image compression. In the proposed model, image grouping, pulse modulation, prediction and encoding operations are performed serially for the transformation of the input to its required output. Image grouping entails the analysis of the details of the images while pulse modulation is used to digitally represent the image by sampling it at regular intervals while each sample is made proportional to the amplitude of the modulating signal. Prediction is a pre-coding operation for examining the pixel stream.

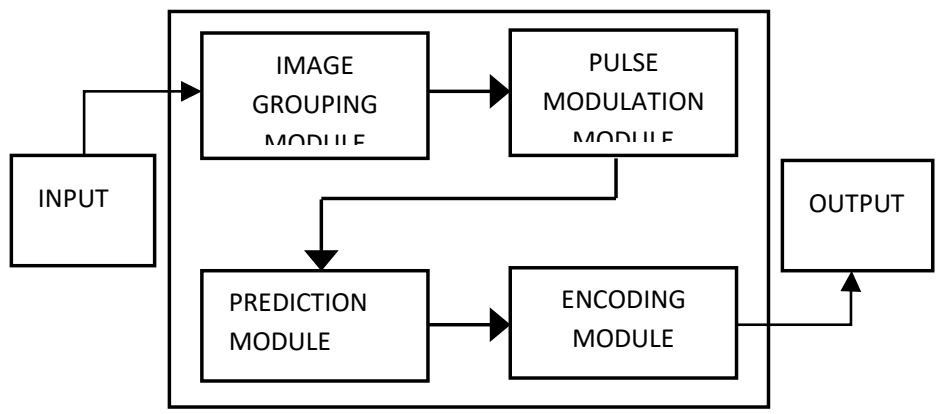

Figure 1: System Architecture

\subsection{Lossless JPEG Coding Algorithm}

The lossless JPEG coding process employs a simple predictive DPCM model (shown in Figure 2) in which the prediction of the sample values are based on estimation from the neighbouring pre-coded reference images in the image [27]. The model encodes the differences between the predicted samples rather than separate encoding of each sample. The basic steps of the lossless operation are shown in Figure 3. The predictor combines up to four neighbouring samples $P, Q, R$ and $S$ shown in the Figure 4 to produce a sample prediction value, $\rho$ at the position labelled $\mathrm{C}$ based on the formula:

$$
\rho=P_{\alpha}+Q_{\beta}+R_{\gamma}
$$

$\alpha, \beta$ and $\beta$ are the weights assigned for pixels $\mathrm{P}, \mathrm{Q}$ and $\mathrm{R}$ respectively. The error of prediction $\varepsilon$, is derived as follows:

$$
\varepsilon=v-\rho
$$

$v$ is the prediction residual (error signal) conditioned for $\mathrm{C}$. 


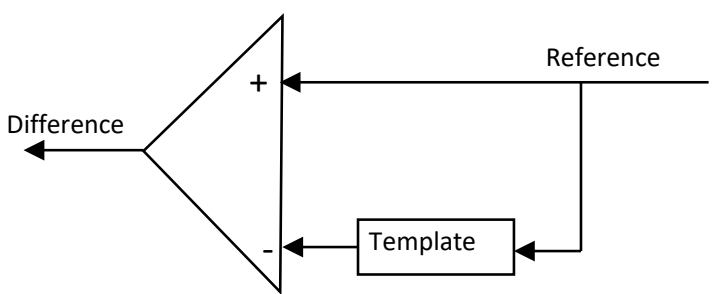

Figure 2: DPCM Encoder

Lossless Encoder

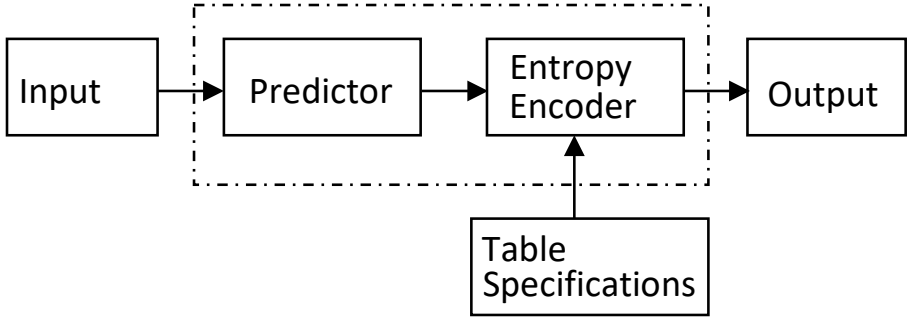

Figure 3: Steps JPEG Lossless Operation

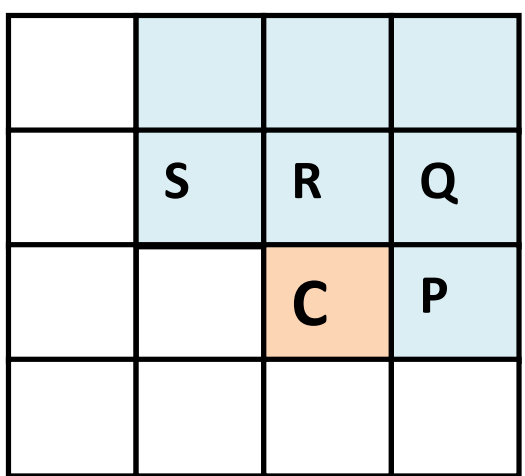

Figure 4: Prediction Process

The brightest operational value with no loss of generality for the predictive function is $P+Q+R$. When $Q$ is the brightest, the maximum of $P$ and $R$ joins $Q$ at the edge, while the minimum of $P$ and $Q$ joins $C$ on the other edge, thus min $(P, R)$ forms a good prediction. The predictive scheme presented in Table 1 is based on the following formula:

$$
\rho=\left\{\begin{array}{lr}
\min (P, R), & \text { if } Q \geq \max (P, R) \\
\max (P, R), & \text { if } Q \leq \min (P, R) \\
P+R-Q, & \text { Otherwise }
\end{array}\right.
$$

The assigned probability distribution $\sigma$, which is the number of information (bits) contained in the image is derived from:

$$
\sigma=\log \left(\frac{1}{\varepsilon_{p}}\right)
$$


Table 1: Prediction Schemes

\begin{tabular}{|c|c|}
\hline Selection-value & Prediction \\
\hline 0 & No prediction \\
\hline 1 & $\mathrm{P}$ \\
\hline 2 & $\mathrm{R}$ \\
\hline 3 & $\mathrm{Q}$ \\
\hline 4 & $\mathrm{P}+\mathrm{R}-\mathrm{Q}$ \\
\hline 5 & $\mathrm{P}+(\mathrm{R}-\mathrm{Q}) / 2$ \\
\hline 6 & $\mathrm{R}+(\mathrm{P}-\mathrm{Q}) / 2$ \\
\hline 7 & $(\mathrm{P}+\mathrm{R}) / 2$ \\
\hline
\end{tabular}

As shown in Table 1, selection value of 0 means no prediction and it is only applicable to differential coding in the hierarchical mode of operation. Selections 1, 2 and 3 are one-dimensional predictors while selections 4, 5, 6 and 7 are two-dimensional predictors. After the prediction of all samples, the sample differences is obtained and subjected to entropy coding in a lossless fashion using Huffman coding for quality retention and speed efficiency.

\subsection{Huffman Compression Algorithms}

The Huffman algorithm relies on an array of unique characters along with their frequency of occurrences to produce an encoded data [28-29]. Huffman coding compression is an entropy image coding algorithm using lossless compression algorithm in which attempt is made to reduce the compositional bits of the image. Encoding of the input (source image) depends on the variable length code table. The code table $C_{T}$ is uniquely derived from the estimated probability of occurrence for each possible value of the image bits as follows:

$$
C_{T}=\sum_{k=0}^{n-1} \quad \sigma\left(\varepsilon_{k}\right) p\left(\varepsilon_{k}\right)
$$

$\varepsilon_{k}$ is prediction residual gray level, $\sigma\left(\varepsilon_{k}\right)$ is the number of bits for $\varepsilon_{k}, p\left(\varepsilon_{k}\right)$ is the probability that a pixel has a certain value $\left(\varepsilon_{k}\right)$ and $\mathrm{n}$ is the number of grey levels contained in the image. The number of bits $b$ is obtained using the image gray level, $g$ as follows:

$$
b=g C_{T}
$$

The entropy coding value $h$, is obtained from:

$$
h=\sum_{k=0}^{n-1} \quad p\left(\varepsilon_{k}\right) \log p\left(\varepsilon_{k}\right)
$$

Image compression then goes through the steps of sorting probabilities per symbol, extraction and combination of the two lowest probabilities and repetition of these two steps until only one probability remains.

\subsection{Lossless JPEG-Huffman Algorithm}

The Lossless JPEG-Huffman algorithm integrates the lossless JPEG and Huffman algorithms to allow further compression of the image while retaining all valuable properties. The flowchart of the visual sequence of activities of the integration is presented in Figure 5. 


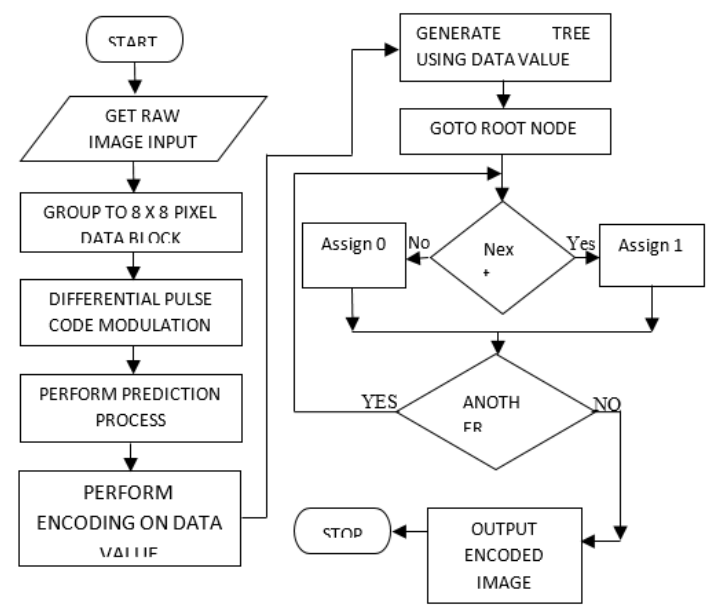

Figure 5: The flowchart of the Lossless JPEG-Huffman Compression Algorithm

Major activities include grouping of the image into $8 \times 8$ pixel blocks, obtaining the DPCM, performing the prediction based on the values presented in Table 1 and then encoding the data values using the Huffman encoding algorithm. This is followed by the listing of all the data values along with their frequencies as nodes. The two nodes with the lowest probabilities are taken and if there is a tie, a random selection amongst the equal frequencies is done. A new node is then constructed out of the selected node while two other nodes are developed as their children. The new node is assigned the sum of the frequencies of its children. The process of combining two nodes of lowest frequencies persists until only one node exists. 0 and 1 are then assigned to the left and right branches respectively and repetitively down the left and right sub-trees. The root to the character leaf node is transverse along the path to obtain the required value.

\section{Experimental Study}

The experimental study of the proposed model was carried out on a Personal Computer (PC) with 4GB RAM, $500 \mathrm{~GB} H D D$ and $2.2 \mathrm{GHz}$ Core i3 Processor running on Windows 10 Operating System. Visual Basic, Dot Net Framework and Visual Studio served as the frontends. Digital Camera (DC) and Drone served as image capturing peripherals. The study was carried out using standard tested imagery as well as camera and geographical information system (GIS) images. The standard images from National Imagery Testing Format (NITF) and Signal and Image Processing Institute (SIPI) databases of digitized images were of various lossless formats which includes tagged images file format (TIFF), picture natural graphics (PNG) and raw binary format. These images contain some checksum values for checking the corruption levels of the images. The GIS images were obtained using a DJI Mavro Drone with the specifications presented in Table 2. The camera images were also obtained based on the specifications presented in Table 3 while the properties of twenty (20) experimental images (shown in Figure 6) from the various sources are listed in Table 4. 
Table 2: Drone specifications

\begin{tabular}{|l|l|l|}
\hline S/NO & Parameter & Specification \\
\hline 1 & Size & $\begin{array}{l}\text { Height: } 83 \mathrm{~mm} \text {; Width: } 83 \mathrm{~mm} ; \\
\text { Length: } 198 \mathrm{~mm}\end{array}$ \\
\hline 2 & Diagonal size & $335 \mathrm{~mm}$ \\
\hline 3 & Maximum ascent speed & $16 \mathrm{feets}$ by $5 \mathrm{~m} / \mathrm{s}$ \\
\hline 4 & Maximum decent speed & $9.8 \mathrm{feets}$ by $3 \mathrm{~m} / \mathrm{s}$ \\
\hline 5 & Flight time & $21 \mathrm{minutes}$ \\
\hline 6 & Maximum travel distance & $13 \mathrm{~km}$ \\
\hline 7 & Sensor pixels & $12.71 \mathrm{~m}$ \\
\hline 8 & Photo sensitivity & $100-1600$ \\
\hline 9 & Shutter speed & Electronic \\
\hline 10 & Modes & Still photography \\
\hline 11 & Exposure bracketing & Auto \\
\hline 12 & File formats & Raw \\
\hline
\end{tabular}

Table 3: Camera specifications

\begin{tabular}{|l|l|l|}
\hline S/NO & Parameter & Specification \\
\hline 1 & Model & Nikon D7000 \\
\hline 2 & Resolution & $23.6 \mathrm{~nm}$ X $15.6 \mathrm{~nm}$ \\
\hline 3 & Sensor & $16 \mathrm{feets}$ by $5 \mathrm{~m} / \mathrm{s}$ \\
\hline 4 & pixel size & $4.7 \mu \mathrm{m}$ \\
\hline 5 & photo sensitivity & $100-6400$ \\
\hline 6 & shutter speed range & 30 seconds to $1 / 8000$ seconds \\
\hline 7 & file formats & enhanced built-in raw \\
\hline 8 & Support Mode & GPS \\
\hline
\end{tabular}

Table 4: Properties of selected images

\begin{tabular}{|l|l|c|l|l|}
\hline Image & Dimension & $\begin{array}{c}\text { Gray Level } \\
\text { (bits/pixel) }\end{array}$ & Source & Size (kb) \\
\hline Lena & $512 \times 512$ & 8 & NITF (Tiff) & 768 \\
\hline Baboon & $512 \times 512$ & 8 & NITF (Tiff) & 768 \\
\hline Barbara & $512 \times 512$ & 8 & NITF (Tiff) & 768 \\
\hline Peppers & $512 \times 512$ & 8 & SIPI (Tiff) & 256 \\
\hline Flowers & $512 \times 512$ & 8 & SIPI (Tiff) & 768 \\
\hline Index & $2048 \times 1680$ & 8 & Camera (Nef) & 1024 \\
\hline Index 2 & $512 \times 512$ & 8 & Camera (Nef) & 768 \\
\hline Index 3 & $2048 \times 1680$ & 8 & Camera (Nef) & 1024 \\
\hline Med 1 & $512 \times 512$ & 8 & NITF (png) & 409 \\
\hline Med 2 & $512 \times 512$ & 8 & NITF (png) & 511 \\
\hline Med 3 & $512 \times 512$ & 8 & NITF (png) & 457 \\
\hline Med 4 & $1280 \times 1024$ & 8 & NITF (png) & 436 \\
\hline Image 1 & $512 \times 512$ & 8 & Drone (Tiff) & 857 \\
\hline Image 2 & $512 \times 512$ & 8 & Drone (Tiff) & 771 \\
\hline Image 3 & $512 \times 512$ & 8 & Drone (Tiff) & 969 \\
\hline Image 4 & $512 \times 512$ & 8 & Drone (Tiff) & 865 \\
\hline Image 5 & $512 \times 512$ & 8 & Drone (Tiff) & 821 \\
\hline Image 6 & $512 \times 512$ & 8 & Drone (Tiff) & 901 \\
\hline Image 7 & $512 \times 512$ & 8 & Drone (Tiff) & 912 \\
\hline Image 8 & $512 \times 512$ & 8 & Drone (Tiff) & 896 \\
\hline
\end{tabular}




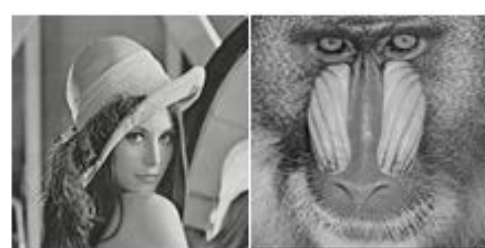

LENA

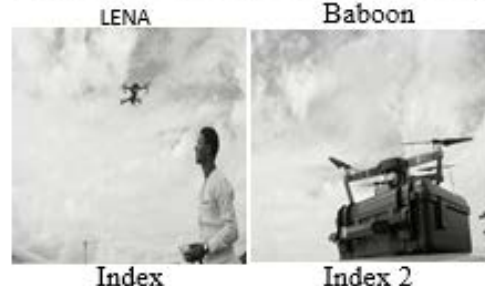

Index

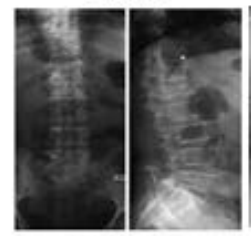

Med 3

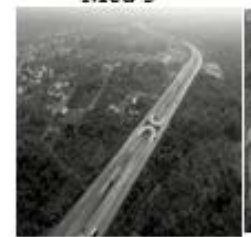

Image 4

Med 4

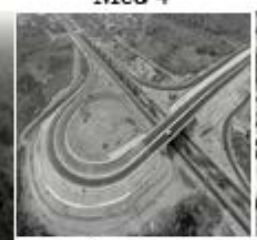

Image 5

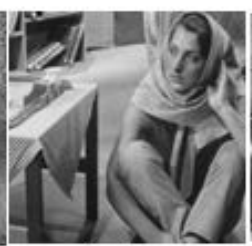

Barbara

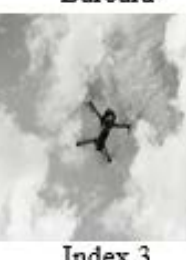

Index 3

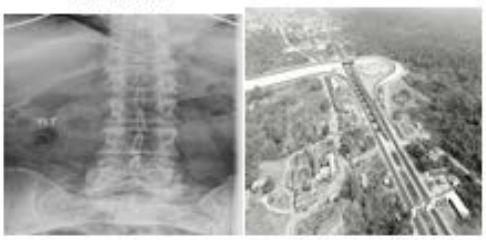

Image 1

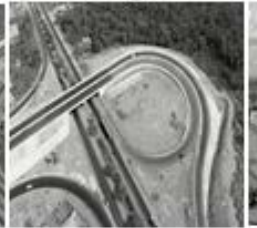

Image 6 (tiff)

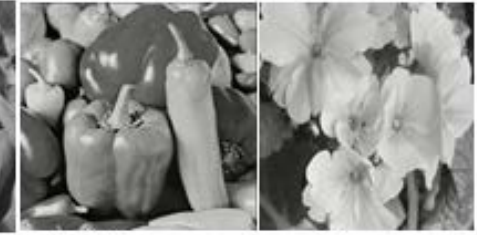

Peppers Flowers

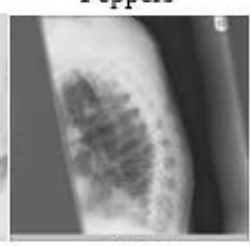

Med 1

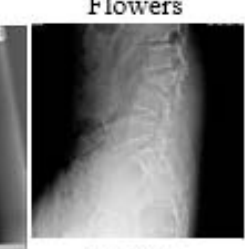

Med 2

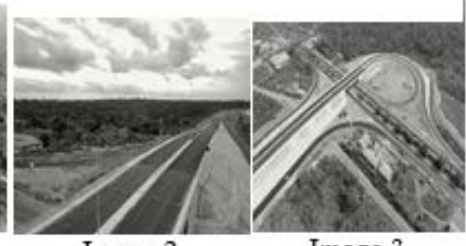

Image 2

Image 3

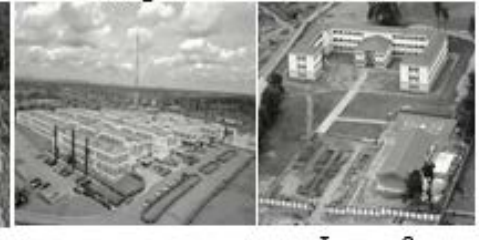

Image 7 (tiff)

Image 8

Figure 6: Experimental images

Prior to compression using lossless jpeg and Huffman algorithms, the images were first subjected to linearization which is conversion into a one-dimensional sequence with no effect on the coding schemes or frequencies. The attribute values for the adopted row-major, diagonal, snake-like and spiral scan linearization schemes are shown in Table 5.

Table 5: Comparison of different linearization schemes

\begin{tabular}{|l|c|c|c|c|c|}
\hline Image & Row & Diagonal & Snake & Spiral & Modality \\
\hline GIS & 1.64 & 1.62 & 1.65 & 1.70 & 8 \\
\hline Camera & 1.73 & 1.64 & 1.73 & 1.69 & 3 \\
\hline Standard tested & 1.18 & 1.18 & 1.17 & 1.18 & 9 \\
\hline
\end{tabular}

Table 6 presents the experimental results for Lossless JPEG-Huffman and Huffman algorithms on the twenty experimental images. The obtained bits per pixel (bpp) values and the compression times are also presented. Results for all the image show higher compression ratios for the integrated algorithm (Lossless JPEG-Huffman) compared to the adapted Huffman algorithm. A comparison of the bits per pixels values in Tables 4 and 6 revealed that the integrated algorithm significantly reduced the bits for every pixel in all the images. It is equally shown that the integrated model exhibit higher compression ratio and PSNR values compared to the lossless compression models for the University of Southern California and Signal and Image Processing Institute (USC-SIPI) and National Imagery Testing Formats (NITF) which have compression ratio and PSNR values of 1.1 and 40 respectively [30]. 
Table 6: Compression ratio for the images

\begin{tabular}{|l|c|c|c|c|c|}
\hline \multicolumn{1}{|c|}{ Image } & $\begin{array}{c}\text { Size } \\
(\mathrm{kb})\end{array}$ & $\begin{array}{c}\text { Lossless JPEG- } \\
\text { Huffman Algorithm } \\
(\mathrm{JPH})\end{array}$ & $\begin{array}{c}\text { Huffman } \\
\text { Algorithm }\end{array}$ & $\begin{array}{c}\text { JPH Bits } \\
\text { per pixel } \\
(\mathrm{bpp})\end{array}$ & $\begin{array}{c}\text { Time } \\
(\mathrm{s})\end{array}$ \\
\hline Lena & 768 & 1.58 & 1.18 & 5.06 & 1.41 \\
\hline Baboon & 768 & 1.61 & 1.21 & 4.97 & 1.42 \\
\hline Barbara & 768 & 1.46 & 1.29 & 5.48 & 1.41 \\
\hline Peppers & 256 & 1.52 & 1.33 & 5.25 & 1.41 \\
\hline Flowers & 768 & 1.51 & 1.31 & 5.30 & 1.41 \\
\hline Index & 1024 & 1.74 & 1.19 & 4.61 & 1.80 \\
\hline Index 2 & 768 & 1.82 & 1.41 & 4.38 & 1.58 \\
\hline Index 3 & 1024 & 1.97 & 1.23 & 4.06 & 1.78 \\
\hline Med 1 & 409 & 1.42 & 1.48 & 5.63 & 1.41 \\
\hline Med 2 & 511 & 1.39 & 1.17 & 5.76 & 1.41 \\
\hline Med 3 & 457 & 1.42 & 1.13 & 5.64 & 1.41 \\
\hline Med 4 & 436 & 1.49 & 1.19 & 5.38 & 1.41 \\
\hline Image 1 & 857 & 1.61 & 1.31 & 4.97 & 1.74 \\
\hline Image 2 & 771 & 1.59 & 1.28 & 5.03 & 1.76 \\
\hline Image 3 & 969 & 1.39 & 1.13 & 5.75 & 1.76 \\
\hline Image 4 & 865 & 1.46 & 1.24 & 5.48 & 1.74 \\
\hline Image 5 & 821 & 1.46 & 1.27 & 5.48 & 1.76 \\
\hline Image 6 & 901 & 1.41 & 1.21 & 5.67 & 1.76 \\
\hline Image 7 & 912 & 1.43 & 1.15 & 5.60 & 1.76 \\
\hline Image 8 & 896 & 1.38 & 1.13 & 5.80 & 1.76 \\
\hline
\end{tabular}

The charts of the pre and post-compression MD, MSE, RMSE, PSNR, AD and SC values are presented in Figures 7 to 12 . Visual inspection of the charts reveals smaller post-compression values for all the metrics. This established the significant contribution of the integrated algorithm in bit size reduction and quality improvement of digital images.

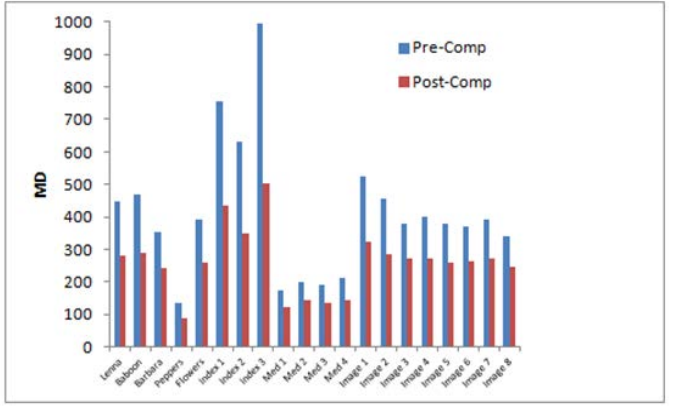

Figure 7: Pre and Post Compression MD values

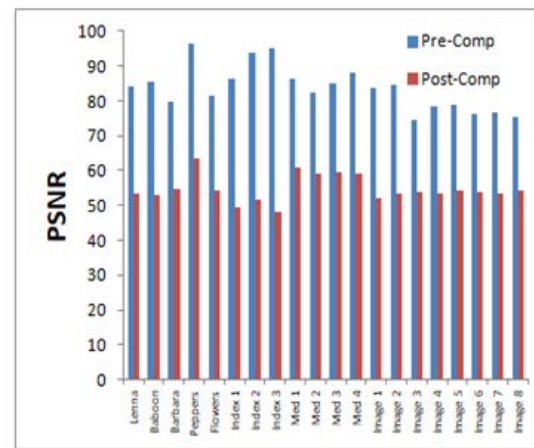

Figure 9: Pre and Post Compression PSNR values

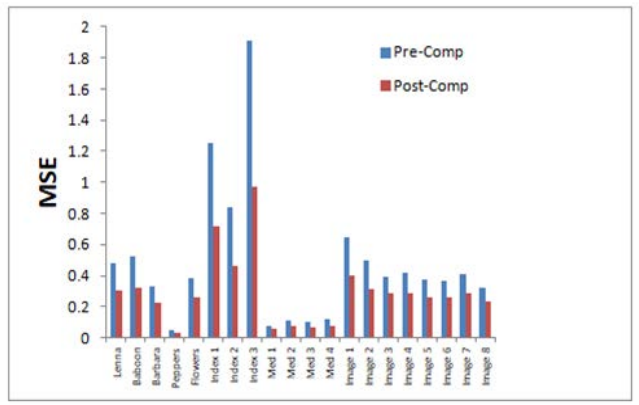

Figure 8: Pre and Post Compression MSE values

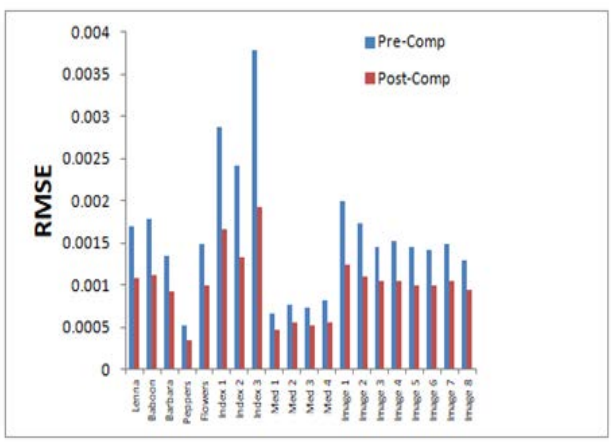

Figure 10: Pre and Post Compression RMSE values 


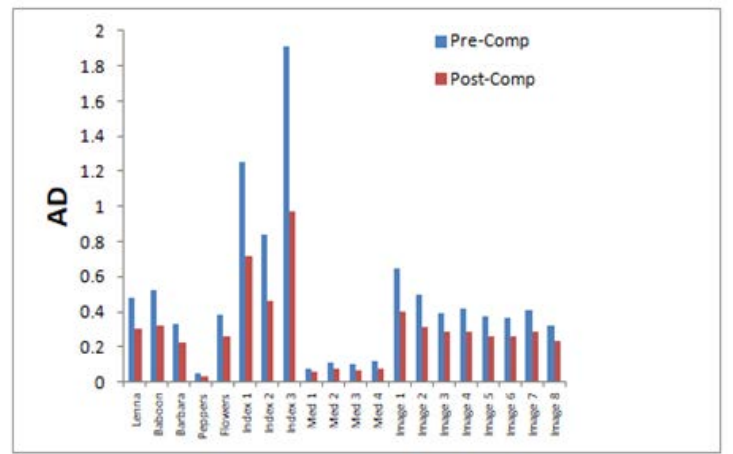

Figure 11: Pre and Post Compression AD values

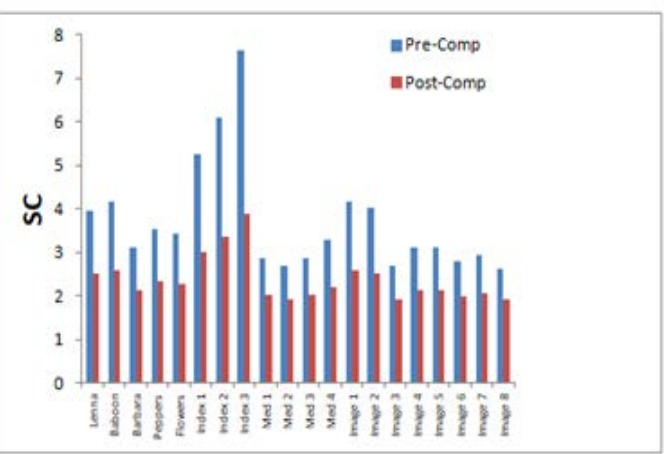

Figure 12: Pre and Post Compression PSNR values

\section{Conclusion}

The integration of lossless JPEG and Huffman algorithms for digital image compression had been presented. The integrated model was implemented and tested with digital images of different sizes, sources and formats. The performance of the proposed model was investigated based on image of different sizes and qualities. Experimental values obtained for standard metrics such as maximum difference, mean square error, peak signal to noise ratio, average difference and structural content were also used to establish the model's practical functions and suitability. The analyses of the obtained experimental values show that the integrated model exhibit superior compression ratios and metric values compared to the standard lossless compression models for the University of Southern California, Signal and Image Processing Institute (USC-SIPI) and National Imagery Testing Formats (NITF) which have standard compression ratio of 1.1 and PSNR of 40 . The suitability of the integrated model for alleviating the problems of bit and size overflow as well as lengthened transmission time through significant reduction of the bit size and redundancies was also established. Further research focuses on combination of other lossless compression algorithms for attaining much more improved compressions. Focus will also be on the compression of 3-D images.

\section{REFERENCES}

[1] H. Kaur, "Data compression techniques in Wireless Sensor Networks", Future Generation Computer Systems, 2016, vol. 64, no. 3, pp: 151-162. http://10.1016/j.future.2016.01.015

[2] M. C. Stamm and K. J. R. Liu, "Anti-Forensics of Digital Image Compression", IEEE Transactions on Information Forensics and Security, vol. 6, no. 3, 2011, vol. 4, no. 1, pp. 1050-1065. http://doi.org/10.1109/TIFS.2011.2119314

[3] C. Guillemot, G. Plonka-Hoch, T. Pock and J. Weickert, "Inpainting-Based Image Compression”, Dagstuhl Reports, 2016, vol. 6, no. 11, pp. 90-107, http://doi.org/10.4230/DagRep.6.11.90

[4] A. Deever, M. Kumar and B. Pillman, "Digital Camera Image Formation: Processing and Storage", Springer Science and Business Media New York, 2013, http://dx.doi.org/10.1007/978-1-4614-07577_2

[5] R. Challa, V. Kumari and P. Sruthi, "Proficient LWE-based Encryption using CAN Compression Algorithm", Proceedings of International Conference on Power, Control, Communication and Computational Technologies for Sustainable Growth, IEEE 2015, pp. 304-307. http://dx.doi.org/10.1109/PCCCTSG.2015.7503951 
Advances in I mage and Video Processing; Volume 7, No. 1, February 2019

[6] S. Shawal, M. Shoyab and S. Begum, "Fundamentals of Digital Image Processing and Basic Concept of Classification", International Journal of Chemical and Process Engineering Research, 2014 Vol. 1, No. 6, pp. 98-108. http://doi.org/10.18488/journal.65/2014.1.6/65.6.98.108

[7] S. E. Umbaugh, "Computer Imaging: Digital Image Analysis and Processing", CRC Press, Boca Raton, Florida, 2005. https://doi.org/10.1259/bjr.79.942.540

[8] M. Petrou and P. Bosdogianni, "Image Processing: The Fundamentals", John Wiley \& Sons, United Kingdom, 1999. http://dx.doi.org/10.1002/9781119994398

[9] E. R. Dougherty, and R. A. Lotufo, "Hands-on Morphological Image Processing", International Society for Optical Engineering, Bellingham Washington, 2003. http://dx.doi.org/10.1117/3.501104

[10] A. F. Frangi, "Simulation and Synthesis in Medical Imaging", IEEE Transactions on Medical Imaging, 2018, Vol. 37, No. 3. http://doi.org/10.1109/TMI.2018.2800298

[11] S. Sachdeva and R. Kaur, "A Review on Digital Image Compression Techniques", International Journal on Recent and Innovation Trends in Computing and Communication, 2014, vol. 2, no. 7. Available: https://www.academia.edu/9220887/A_Review_on_Digital_Image_Compression_Techniques, Accessed 15/03/2017

[12] M.A. El-Sharkawy ; C.A. White ; H. Gundrum, "Image Compression Using Wavelet Transform and Vector Quantization", Proceedings of the 39th Midwest Symposium on Circuits and Systems,, Ames, IA, USA, 21 Aug. 1996. http://dx.doi.org/10.1109/MWSCAS.1996.587822

[13] Y. E. Gelogo and T. Kim, "Compressed Images Transmission Issues and Solutions", International Journal of Computer Graphics, 2014, vol.5, no.1, pp.1-8. http://dx.doi.org/10.14257/ijcg.2014.5.1.01

[14] D. Shapira and A. Daptardar, "Adapting the Knuth-Morris-Pratt Algorithm for Pattern Matching in Huffman Encoded Texts", Information Processing and Management, 2006, vol. 42, no. 2, pp. 429-439. http://dx.doi.org/10.1016/j.ipm.2005.02.003

[15] A. Alarabeyyat, S. Al-Hashemi, T. Khdour, S. Bani-Ahmad, M. Hjouj and R. Al-Hashem, "Lossless Image Compression Technique Using Combination Methods", Journal of Software Engineering and Applications, 2012, vol. 5, pp. 752-761. http://dx.doi.org/10.4236/jsea.2012.510088

[16] M. Ailenberg, O. Rotstein, "An improved Huffman coding method for archiving text, images, and music characters in DNA", Biotechniques. 2009, vol. 47, no. 3, pp 747-54. http://doi.org/10.2144/000113218

[17] N. E. Malandrakis, "Error Prediction for Speech Recognition using Acoustic and Linguistic Cues", unpublished Thesis on Degree Programme in Electronics and Computer Engineering at Technical University of Crete Chania, Greece, 2007. Available: https://sail.usc.edu/ malandra/files/thesis.pdf, Accessed 23/05/2017

[18] S. Singh and P. Pandey, "Enhanced LZW Technique for Medical Image Compression", Proceedings of $3^{\text {rd }}$ International Conference on Computing for Sustainable Global Development, IEEE, 2016. Available: https://ieeexplore.ieee.org/document/7724428, Accessed 23/05/2017

[19] N. Bansal and K. Dubey, "Image Compression Using Hybrid Transform Technique”, Journal of Global Research in Computer Science, 2013, vol. 4, no. 1, pp. 13-17. Available: http://www.rroij.com/openaccess/image-compression-using-hybrid-transform-technique-13-17.pdf, Accessed 23/07/2017 
[20] S. Kumar, M. Rawat, V. Gupta and S. Kumar, "The Novel Lossless Text Compression Technique Using Ambigram Logic and Huffman Coding", Journal of Information and Knowledge Management, 2012, vol. 2 2, no. $2 . \quad$ Available: https://pdfs.semanticscholar.org/a2c1/549bae6fb1c215ff8446dd060b8351b66935.pdf, Accessed $16 / 05 / 2015$

[21] M. Talu and I. Turkoglu, "Hybrid Lossless Compression Method for Binary Images", Allan Institute for Artificial Intelligence, 2010, Available: https://pdfs.semanticscholar.org/0358/70a8576e986840b09499125114e23dd57839.pdf, Accessed $25 / 03 / 2016$

[22] A. Alarabeyyat, S. Al-Hashemi, T. J. Khdour, M. H. Btoush, S. Bani-Ahmad and A. Rafeeq, "Lossless Image Compression Technique Using Combination Methods", Journal of Software Engineering and Applications, 2010, vol. 5, no. 10. https://doi.org/10.4236/jsea.2012.510088

[23] P. Howard and J. Vitter, "Fast and Efficient Lossless Compression", IEEE Computer Society, 1993, pp. 351-360. http://dx.doi.org/10.1109/DCC.1993.253114

[24] R. N. Shrikhande and V. K. Bairagi, "Comparison of Different Methods for Lossless Medical Image Compression”, Global Journal of Engineering, Design and Technology, 2013, vol. 2, no. 3, pp. 36-40, Available: https://www.longdom.org/articles/comparison-of-different-methods-for-losslessmedical-image-compression.pdf, Accessed 18/09/2017

[25] K. Pattanaik and K. Mahapatra, "A lossless image compression technique using simple arithmetric operations and its FPGA implementation", Department of Electronics and Communication Engineering, NIT Rourkela, India. IEEE, 2006, pp. 1-6. Available: https://www.researchgate.net/publication/224713063_A_Lossless_Image_Compression_Technique _using_Simple_Arithmetic_Operations_and_its_FPGA_Implementation, Accessed 23/04/2016

[26] R. Vanaja, N. Prabha and N. Stalin, "Efficient Architecture for SPIHT Algorithm in Image Compression", International Journal of Advanced Research in Computer Science Engineering and Information Technology, 2013, vol. $1, \quad$ no. $3 . \quad$ Available: https://pdfs.semanticscholar.org/80a1/a5dc1a2948fb86b80bd7fe8c56be93301e68.pdf, Accessed $16 / 08 / 2016$

[27] W. Pennebaker and J. Mitchell, "JPEG Still Image Data Compression Standard", New York: Van Nostrand Reinhold, 2003. https://dl.acm.org/citation.cfm?id=573326

[28] D. Huffman, "A Method for the construction of Minimum-redundancy Codes", Proceedings of IRE, vol. 40, no.10, pp. 1098-1101. http://dx.doi.org/10.1109/JRPROC.1952.273898

[29] M. Shikhar, "Greedy Algorithms Set 3 Huffman Coding", 2017, Available: http://www.geeksforgeeks.org/greedy-algorithms-set-3-huffman-coding/. Acessed 15/12/2016

[30] A. G. Weber, "USC-SIPI Image Database Version 6", USC Viterbi School of Computing, 2018, Available: www.sipi.usc.edu, Accessed 23/08/2016 